\title{
ARTICLE
}

\section{Study of the direct response of a monolithic silicon telescope to charged particles at different energies}

\author{
Stefano Agosteo ${ }^{\mathrm{a}, \mathrm{b}}$, Enrico Borsato ${ }^{\mathrm{c}, \mathrm{d}}$, Flavio Dal Corso ${ }^{\mathrm{c}}$, Alberto Fazzi $^{\mathrm{a}, \mathrm{b}}$, Franco Gonella ${ }^{\mathrm{c}}$, Maria Vittoria Introini ${ }^{\mathrm{a}, \mathrm{b}}$, \\ Michele Lorenzoli $^{\mathrm{a}, \mathrm{b}}$, Matteo Pegoraro ${ }^{\mathrm{c}}$, Andrea Pola ${ }^{\mathrm{a}, \mathrm{b}^{*}}$, Vincenzo Varoli ${ }^{\mathrm{a}, \mathrm{b}}$ and Pierluigi Zotto ${ }^{\mathrm{c}, \mathrm{d}}$ \\ ${ }^{a}$ Politecnico di Milano, Dipartimento di Energia, Sezione di Ingegneria Nucleare, via Ponzio 34/3 20133 Milano, Italy; ${ }^{b}$ INFN, \\ Sezione di Milano, via Celoria 16, 20133 Milano, Italy; ${ }^{c}$ INFN, Sezione di Padova, via Marzolo 8, 35131 Padova, Italy; \\ ${ }^{d}$ Università di Padova, Dipartimento di Fisica, via Marzolo 8, 35131 Padova, Italy
}

\begin{abstract}
An innovative silicon device based on the monolithic silicon telescope was recently presented and investigated for solid state microdosimetry (of neutron field and hadron beams) and for neutron spectrometry. This device consists of a surface $\Delta \mathrm{E}$ stage, about $2 \mu \mathrm{m}$ in thickness, coupled to an E stage, about $500 \mu \mathrm{m}$ in thickness, made out of a single silicon wafer. The $\Delta \mathrm{E}-\mathrm{E}$ device is capable of providing information about the kind and the energy of the impinging particles. This feature can be exploited for an optimized i) tissue-equivalent correction of microdosimetric distributions measured with the silicon device and ii) gamma-neutron discrimination. This work discusses the characterization of the direct response of this detector to charged particles To this aim, a sample device with a sensitive area of about $1 \mathrm{~mm}^{2}$ was irradiated with mono-energetic protons and alpha particles of energy between $1 \mathrm{MeV}$ and $5 \mathrm{MeV}$ at the Van De Graaff $\mathrm{CN}$ accelerator of the Legnaro National Laboratories of the Italian Institute of Nuclear Physics (LNL-INFN). The experimental $\Delta \mathrm{E}-\mathrm{E}$ scatter plots were analyzed in details and compared with results of an analytical model. The agreement between the results was satisfactory for events distributed over the theoretical $\Delta \mathrm{E}-\mathrm{E}$ curve. Events of the experimental scatter-plots characterized by a linear correlation between energy deposited in the $\Delta \mathrm{E}$ and in the $\mathrm{E}$ detector highlighted were attributed to channeling effect.
\end{abstract}

\section{Keywords: monolithic silicon telescope; silicon detector; ion beam}

\section{Introduction}

Feasibility studies proposed recently by Agosteo et al. demonstrated the possibility of using a monolithic silicon telescope (MST) for solid state microdosimetry (of neutron field and hadron beams) [1] and for neutron spectrometry [2]. The device, in principle a monolithic $\Delta \mathrm{E}-\mathrm{E}$ detector, demonstrated to be capable of providing information about the kind and the energy of the impinging particles. This feature was exploited for an optimized tissue-equivalent correction of microdosimetric distributions measured with the MST [1] and gamma-neutron discrimination [2].

The MST can be used for i) solid state microdosimetry by coupling it with a tissue-equivalent converter or ii) for neutron spectrometry by placing in front of it a polyethylene converter. In both cases, the silicon device measured the energy deposited in his active volume by secondary charged particles generated in the converter.

In order to characterize the direct response of this detector to charged particles, a device with a sensitive

*Corresponding author. Email: andrea.pola@polimi.it area of about $1 \mathrm{~mm}^{2}$ was irradiated with protons and alpha particles of energy between $1 \mathrm{MeV}$ and $5 \mathrm{MeV}$ at the Van De Graaff $\mathrm{CN}$ accelerator of the Legnaro National Laboratories of the Italian Institute of Nuclear Physics (LNL-INFN).

\section{Materials and methods}

The MST is a semiconductor device, developed in collaboration with ST-Microelectronics, constituted by a surface $\Delta \mathrm{E}$ detector, about $2 \mu \mathrm{m}$ in thickness, coupled to a residual energy measurement detector E, about $500 \mu \mathrm{m}$ in thickness (Figure 1). The two detectors are made out of a single silicon wafer by a high energy ion implantation. This fabrication process generate a deep electrode which acts as a "watershed" for the collection of charge produced in the device by impinging radiation. The structure of the $\Delta \mathrm{E}-\mathrm{E}$ device, in particular the presence of the $\mathrm{E}$ stage below the $\Delta \mathrm{E}$ stage, offers the opportunity of measuring event by event the residual energy of particles with range lower than about $500 \mu \mathrm{m}$.

The characterization was performed by irradiating directly a sample MST $1 \mathrm{~mm}^{2}$ in sensitive area with 
mono-energetic protons and alpha particles of energy between $1 \mathrm{MeV}$ and $5 \mathrm{MeV}$ at the Van De Graaff $\mathrm{CN}$ accelerator of the Legnaro National Laboratories of the Italian Institute of Nuclear Physics (LNL-INFN).

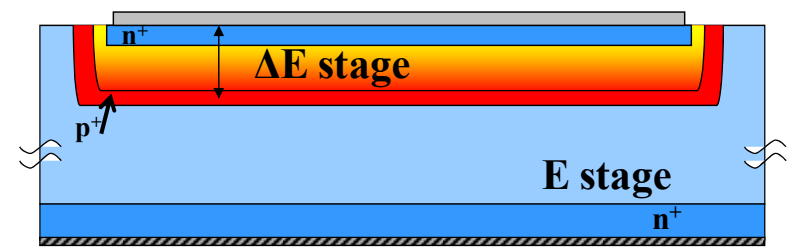

Figure 1. Sketch of the monolithic silicon telescope, constituted by a surface $\Delta \mathrm{E}$ detector, about $2 \mu \mathrm{m}$ in thickness, coupled to a residual energy measurement detector $\mathrm{E}$, about $500 \mu \mathrm{m}$ in thickness. These two stages are separated by a $\mathrm{p}^{+}$ electrode obtained by high energy ion implantation.

The device was placed within an irradiation chamber (Figure 2a) designed for testing the silicon detector (Figure 2b) and for measuring the beam current at the detector surface [3]. At each measurement, the beam current was firstly set-up by mean of a three collimator system (C1, C2 and C3 in Figure 1a), a beam stopper (C4 in Figure 2a) and an alignment cross (Figure 2b). The collimator block is followed by a removable beam stopper (C4) made of a $0.25 \mathrm{~cm}$ tantalum foil which intercepts the collimated beam and is used for tests and calibration. Each collimator and the beam stopper are readout independently. The last block (Figure $2 \mathrm{~b}$ ) is put on a movable arm and is made of two pieces: the detector to be tested and an alignment system composed by four tantalum quadrants $\mathrm{Q}$, each one readout independently, delimiting a cross shaped region. The set-up procedure is the following. When beam is on, the beam stopper and the alignment cross are put on the beam trajectory. Then $\mathrm{C} 4$ is removed, once verified that the beam really hits it, so that the beam hits the alignment cross. The beam is aligned when the current readings of each tantalum quadrant is about equal, that is when moving the cross by one step the beam is mainly within one of the tantalum elements instead of being shared among all four elements. This operation is fast and easy using a well focused beam with a current around $300-400 \mathrm{pA}$ on $\mathrm{C} 4$. The detector is then moved to the measurement position applying the known displacement with respect to $\mathrm{Q}$. The precision of the positioning system is around $0.5 \mathrm{~mm}$.

This operation allowed to maintain counting rates at acceptable values (a few kcps) and to minimize radiation damage.

The two-stages detector was connected to a custom front-end electronics placed in the irradiation chamber.

\section{Results and discussion}

The silicon device was placed within the irradiation chamber and irradiated with protons and alpha particles. The signals generated by the impinging radiation in the
$\Delta \mathrm{E}$ and $\mathrm{E}$ detectors were amplified and shaped by two independent electronic chains and collected by a custom 2-channel ADC in coincidence mode in order to maintain the time correlation. The $\Delta \mathrm{E}-\mathrm{E}$ pairs were acquired event-by-event and processed to have $\Delta \mathrm{E}-\mathrm{E}$ scatter-plots.

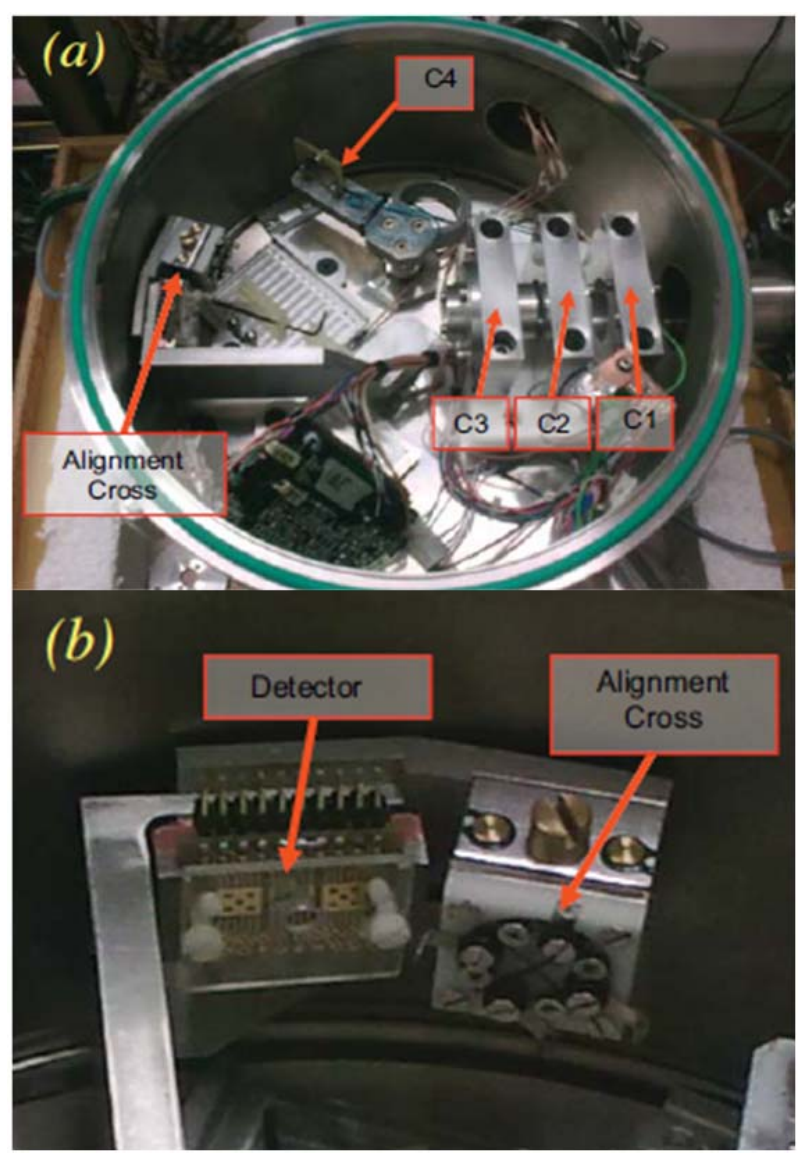

Figure 2. Picture of the irradiation chamber (a) and of the detector holder (b).

To reproduce and analyze the response of the monolithic silicon telescope to charged particle an analytical model was developed. This model, described in details by Agosteo and Pola [4], takes into account the actual geometrical structure of the device $(0.24 \mu \mathrm{m}$ titanium-based dead layer, $1.9 \mu \mathrm{m} \Delta \mathrm{E}$ stage, $500 \mu \mathrm{m} \mathrm{E}$ stage).

\subsection{Irradiations with mono-energetic protons}

The scatter-plots of the energy deposited in the $\Delta \mathrm{E}$ and in the $\mathrm{E}$ detectors by impinging protons at different energies are shown in Figure 3. In particular, distribution A, B, C, D, E, F and G corresponds to irradiations with protons of nominal energy 1, 2.5, $3,3.5,4,4.5$ and $5 \mathrm{MeV}$, respectively. The vertical spread-out of the measured distributions can be attributed to energy resolution of the $\Delta \mathrm{E}$ detector, mainly determined by an high electronic noise (10 $\mathrm{keV}-\mathrm{FWHM}$ ). Since the resolution of $\mathrm{E}$ detector was about $5 \mathrm{keV}-\mathrm{FWHM}$, the horizontal widening of $\Delta \mathrm{E}-\mathrm{E}$ 
scatter plots is mainly due to the spread-out in the energy distribution of impinging protons due to scatterings.

Figure 3 shows also the curve which describe the theoretical relation between the energy deposited in the $\Delta \mathrm{E}$ detector and that releases in the $\mathrm{E}$ detector obtained through the analytical model.

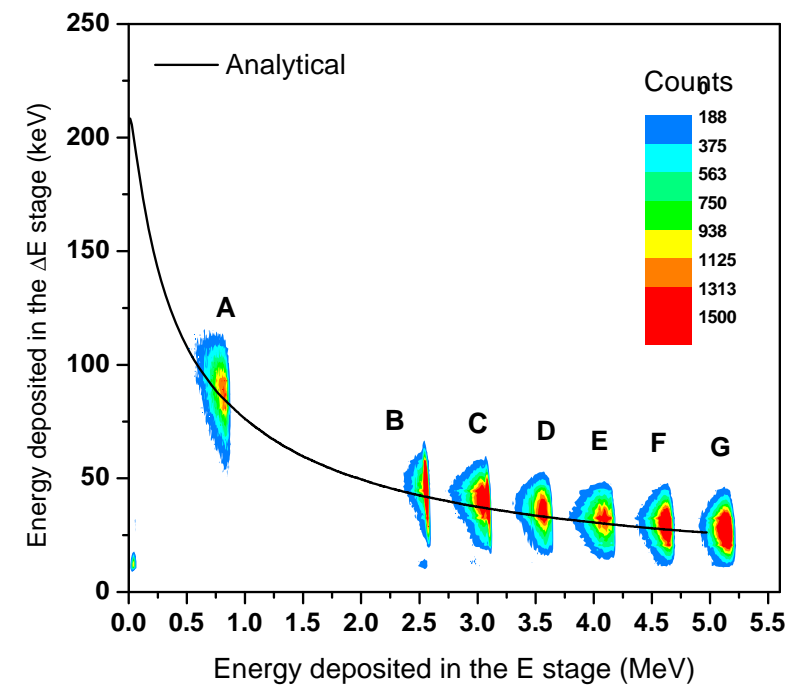

Figure 3. $\Delta \mathrm{E}-\mathrm{E}$ scatter plot obtained by irradiating the monolithic silicon telescope with protons at different energies: distribution A, B, C, D, E, F and G corresponds to irradiations at a nominal energy of $1,2.5,3,3.5,4,4.5$ and $5 \mathrm{MeV}$, respectively.

The agreement between analytical and experimental results demonstrates:

i) the consistency between the nominal and the actual thickness of the $\Delta \mathrm{E}$ detector ;

ii) the absence or negligibility of charge losses at the deep $\Delta \mathrm{E}$-E interface.

\subsection{Irradiation with alpha particles}

Figure 4 shows the $\Delta \mathrm{E}$ scatter plots measured by irradiating the monolithic silicon telescope with alpha particles at different energies. Distributions A-I correspond to irradiations with alpha particles of nominal energy which goes from 1 to $5 \mathrm{MeV}$ with step of $0.5 \mathrm{MeV}$.

The measured $\Delta \mathrm{E}-\mathrm{E}$ scatter plots highlight the presence of:

1) events distributed over the theoretical $\Delta \mathrm{E}$-E curve, due to alpha particles accelerated at an energy lower than the nominal value (scattering in the acceleration channel);

2) events located at lower energies deposited in the $\Delta \mathrm{E}$ detector, but at same time at higher energies deposited in the $\mathrm{E}$ detector with respect to theoretical values.

In particular a linear correlation between the energy deposited in the two detector is evident. As highlighted in Figure 5 for a single measurement (irradiation with 3

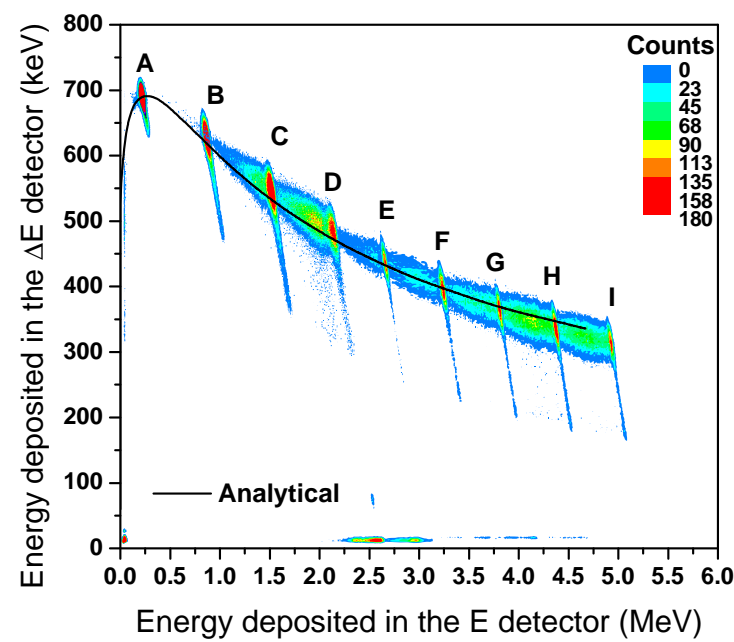

Figure 4. $\Delta \mathrm{E}-\mathrm{E}$ scatter plot obtained by irradiating the monolithic silicon telescope with alpha particles at different energies: distributions A-I correspond to a nominal energy from $1 \mathrm{MeV}$ to $5 \mathrm{MeV}$ with step of $0.5 \mathrm{MeV}$.

$\mathrm{MeV}$ alphas), distributions of events of type 2) lie on lines with unit negative slope. This means that the sum of the energy deposited in the $\Delta \mathrm{E}$ detector and in the $\mathrm{E}$ detector is constant and equal to the energy the impinging particle has at the detector entrance. This population is probably due to channelling of ions into the silicon crystal.

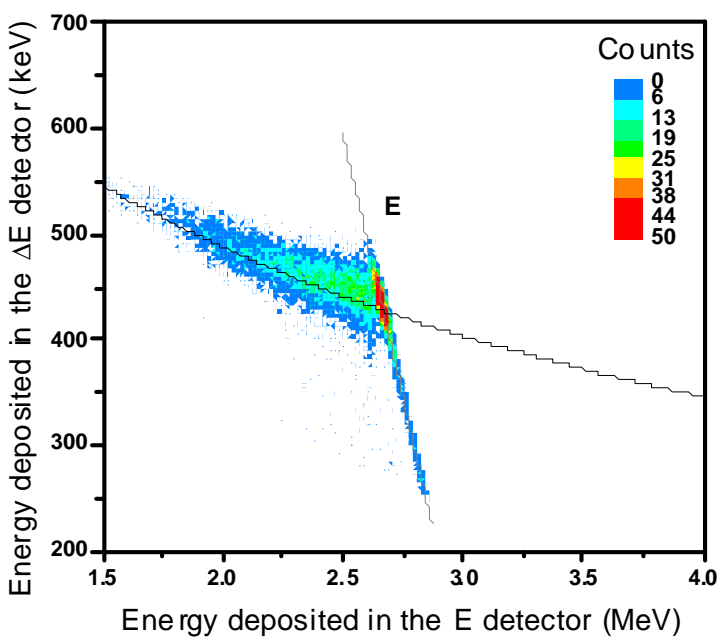

Figure 5. $\Delta \mathrm{E}-\mathrm{E}$ scatter plot obtained by irradiating the silicon telescope with $3 \mathrm{MeV}$ alpha particles. Black curve correspond to the theoretical $\Delta \mathrm{E}-\mathrm{E}$ distribution, while gray line indicates a unit negative slope trend.

\section{Conclusions}

A monolithic silicon telescope $1 \mathrm{~mm}^{2}$ in sensitive area was irradiated with protons and alpha particles of energy between 1 and $5 \mathrm{MeV}$. The measured $\Delta \mathrm{E}$-E scatter plots were compared with results of an analytical model 
which takes into account the actual geometry of the device. Irradiations with protons results demonstrated the consistency between the nominal and the actual thickness of the $\Delta \mathrm{E}$ detector, together with the absence or negligibility of charge losses at the deep $\Delta \mathrm{E}-\mathrm{E}$ interface. Distributions obtained with alpha particles highlighted the presence of events characterized by a linear correlation between energy deposited in the $\Delta \mathrm{E}$ and in the $\mathrm{E}$ detector. These events were attributed to channeling of ions in the silicon crystal. Further measurements are needed to support this interpretation.

\section{References}

[1] S. Agosteo and A. Pola, Silicon microdosimetry, Radiat. Prot. Dosim. 143 (2-4) (2011), pp. 409-415.
[2] S. Agosteo, P. Colautti, J. Esposito, A. Fazzi, M.V. Introini and A. Pola, Characterization of the energy distribution of neutrons generated by $5 \mathrm{MeV}$ protons on a thick beryllium target at different emission angles, Appl. Radiat. Isot. 69 (12) (2011), pp. 1664-1667.

[3] S. Agosteo, E. Borsato, F. Dal Corso, A. Fazzi, F. Gonella, M.V. Introini, I. Lippi, M. Lorenzoli, L. Modenese, F. Montecassiano, M. Pegoraro, A. Pola, V. Varoli and P. Zotto, Performance of a proton irradiation chambre, Nucl. Instr. Meth. Phys. Res. A 664 (2012), pp. 193-202.

[4] S. Agosteo and A. Pola, Analytical model for a monolithic silicon telescope - Response function of the $E$ stage, Rad. Meas. 43 (9-10) (2008), pp. 1487-1492. 